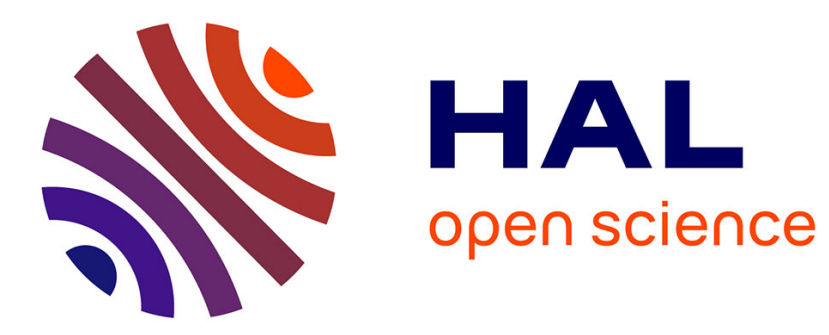

\title{
Composer avec le risque: la frontière sud de l'Albanie entre politique des États et solidarités locales
}

Pierre Sintès, Gilles de Rapper

\section{To cite this version:}

Pierre Sintès, Gilles de Rapper. Composer avec le risque: la frontière sud de l'Albanie entre politique des États et solidarités locales. Revue d'Etudes Comparatives Est-Ouest, 2006, 37 (4), pp.243-271. halshs-00165537

\section{HAL Id: halshs-00165537 https://shs.hal.science/halshs-00165537}

Submitted on 27 Jun 2008

HAL is a multi-disciplinary open access archive for the deposit and dissemination of scientific research documents, whether they are published or not. The documents may come from teaching and research institutions in France or abroad, or from public or private research centers.
L'archive ouverte pluridisciplinaire HAL, est destinée au dépôt et à la diffusion de documents scientifiques de niveau recherche, publiés ou non, émanant des établissements d'enseignement et de recherche français ou étrangers, des laboratoires publics ou privés. 
Article paru dans Revue d'études comparatives Est-Ouest 37 (2006), 4 : 243-271

\author{
COMPOSER AVEC LE RISQUE : \\ LA FRONTIÈRE SUD DE L'ALBANIE ENTRE POLITIQUE DES ÉTATS ET SOLIDARITÉS LOCALES
}

Gilles de Rapper et Pierre Sintès*

Résumé. - Depuis sa création en 1913, la frontière entre la Grèce et l'Albanie peut être vue à la fois comme une zone d'instabilité géopolitique et comme une éventuelle ressource pour les populations vivant à sa proximité. Cet article explore les implications de la notion de risque considérée dans ses deux versants de «péril » et d'« opportunité » et s'interroge sur les réponses, individuelles et collectives, apportées par la population locale, du côté albanais de la frontière, à cette situation de risque. Le fait le plus notable est un processus de fragmentation de la société et de particularisation de certains groupes sur la base de leur relation à la frontière et à la Grèce, qui peut lui-même être vu comme un risque pour l'intégrité de l'État albanais.

\title{
Introduction : frontière et risque
}

[245] Rapprocher la notion de frontière politique de celle de risque revient à parler de l'État. À la fois limite physique et marqueur symbolique de l'exercice du pouvoir par l'État, la frontière est l'un des lieux où ce dernier s'expose à un certain nombre de dangers. Elle est par exemple la première ligne de défense en cas d'agression extérieure, et sa violation est une atteinte à l'intégrité du pays. De la même manière, en tant que zone de transition, les régions frontalières peuvent plus facilement abriter des activités, des réseaux ou des

\footnotetext{
* Respectivement chargé de recherche au C.N.R.S., Institut d'ethnologie méditerranéenne et comparative, Maison méditerranéenne des sciences de l'homme, Aix-en-Provence (derapper@mmsh.univ-aix.fr), et A.T.E.R., École Normale Supérieure, Paris (pierre.sintes@ens.fr).
} 
institutions politiques qui s'opposent à l'État ou limitent l'exercice de sa souveraineté. Enfin, la frontière politique est souvent doublée ou traversée de frontières « culturelles » qui, quand elles ne correspondent pas dans leur tracé aux contours des États, remettent en question l'homogénéité ethnique et culturelle de l'État-nation (Donnan, Wilson 1999, p. 10-11). Pour toutes ces raisons, la notion de risque est, du point de vue de l'État, consubstantielle à celle de frontière.

Dans ce qui suit, nous nous intéressons cependant moins à la gestion de ce « risque de frontière» encouru par les États qu'à la façon dont cet objet géopolitique, dans le contexte moderne des États-nations balkaniques, peut représenter une menace comme impliquer des prises de risque de différentes natures pour les populations vivant à sa proximité. Pour ce faire, la première partie de notre contribution rappelle les différentes situations à risque qui menacent les habitants de la région frontalière gréco-albanaise ${ }^{1}$; la deuxième partie expose les implications des risques de la frontière, en particulier dans le domaine de l'action étatique; la troisième partie porte sur les réponses individuelles et collectives apportées aux différentes formes de risque frontalier.

\section{La frontière gréco-albanaise et le risque}

Parmi les risques inhérents à toute frontière nationale (qu'il s'agisse de "périls » ou «d'opportunités»), la frontière gréco-albanaise en présente deux qui nous paraissent particulièrement marqués : (1) un risque de conflit international, rendu aigu par le caractère disputé de la frontière, et qui s'est plusieurs fois actualisé depuis sa création ; (2) un risque lié aux identifications et catégorisations des individus et des groupes, la frontière (comme limite de catégories nationales) se surimposant ici à une hétérogénéité culturelle préexistante.

[246] Un troisième type de risque ne nous retiendra pas, mais doit être signalé. La frontière gréco-albanaise, comme toutes les frontières balkaniques, se présente, pour reprendre les termes de Lucien Febvre (Febvre 1928), comme « un fossé entre nationalités fortement distinctes » et ne relève pas du modèle des "marches séparantes ", régions désertes faisant tampon entre deux États. La zone frontalière doit donc être constamment occupée et son dépeuplement présente un risque pour l'État. Du côté albanais, la fixation de la population dans les coopératives agricoles à partir des années 1950 et la sédentarisation des pasteurs aroumains vers la même époque semblent avoir écarté le risque de désertification des régions frontalières. Du côté grec, malgré l'installation de réfugiés d'Asie mineure dans les régions frontalières du Nord dans les années 1920, la menace de désertification (départs consécutifs à la guerre civile, exode rural et émigration) s'est suffisamment fait ressentir pour que, en 1960, la région de Kastoria soit déclarée «zone franche» pour le travail de la fourrure, mesure destinée à maintenir son attractivité

\footnotetext{
${ }^{1}$ Bien que des enquêtes de terrain aient été réalisées des deux côtés de la frontière, la présente contribution porte davantage sur le côté albanais de la frontière. Le matériel a été recueilli entre 1995 et 1997 par G. de Rapper et entre 2000 et 2002 par G. de Rapper et P. Sintès.
} 
économique, tandis que, dans les années 1980, une série de mesures économiques visaient à assurer la présence grecque dans les régions frontalières septentrionales, considérées comme sensibles et menacées (Deslondes 1997, p. 71 et 76).

\subsection{Le risque de conflit international}

La notion de risque apparait sur la frontière gréco-albanaise d'abord en raison de son caractère de limite internationale discutée et disputée depuis la date même de sa création Ceci découle de la complexité des appartenances collectives dans une région traversée par un certain nombre de frontières culturelles et ethniques dont les principaux marqueurs sont la langue et l'appartenance confessionnelle.

La frontière est disputée dans son tracé dès la création de l'État albanais. Elle résulte en effet d'un compromis qui ne satisfait personne. Elle laisse de chaque côté des « minorités » irrédentes qui menacent la stabilité et l'existence des États qu'elle conjoint.

La frontière politique, telle qu'elle est tracée en 1913, limite le nouvel État albanais (dont l'indépendance est proclamée à Vlorë le 28 novembre 1912 et reconnue internationalement le 29 juillet 1913) et sanctionne par là l'annexion par le royaume de Grèce des nouveaux territoires du nord, Épire et Macédoine. Elle marque en ce sens le partage des derniers territoires ottomans des Balkans, à l'issue de la Première Guerre balkanique (octobre 1912-mai 1913) et la création d'un État albanais indépendant dont l'existence était contestée par l'ensemble des voisins (Winnifrith 2002).

Le tracé général des frontières du nouvel État était déterminé par les rivalités qui opposaient les grandes puissances. Les Français, les Anglais et les Russes, qui soutenaient plutôt la Grèce et la Serbie, étaient partisans [247] d'une Albanie minimale, alors que les Italiens et les Autrichiens cherchaient à contrer l'influence des trois premiers et de leurs protégés en se faisant les partisans d'une Albanie la plus étendue possible. Le résultat de ce rapport de force est un compromis d'abord atteint en juillet 1913 (Conférence de Londres) et confirmé, à peu de choses près, par la Conférence des Ambassadeurs de novembre 1921. La commission internationale chargée du tracé de la frontière devait prendre en compte la «nationalité » des populations en présence, selon le critère de la langue parlée par les femmes et les enfants, les hommes étant plus susceptibles d'être bilingues en raison de leurs déplacements et de leurs activités professionnelles. Ce travail de terrain se déroula entre octobre et décembre 1913 et fut sanctionné par le Protocole de Florence (décembre 1913). La commission reprit son travail au lendemain de la Première Guerre mondiale (19221925), alors que leur franchissement par les troupes grecques avait remis en question les limites de 1913, et les derniers litiges entre la Grèce et l'Albanie furent réglés en octobre 1924, avec l'évacuation par la Grèce d'un certain nombre de villages de la région de Korçë (Puto 1974).

Cette genèse problématique montre à quel point une conception exclusivement nationale de la frontière n'est pas facilement transposable aux réalités du terrain. Dès sa 
création, cette limite ne pouvait être qu'inadéquate dans la mesure où les appartenances nationales ne dessinaient jamais dans cette région des territoires exclusifs, et où les allégeances des différents groupes n'étaient pas cristallisées, notamment chez les chrétiens orthodoxes albanophones qui pouvaient hésiter entre les nations grecque et albanaise.

La frontière sépare des pays qui, depuis sa création, se sont le plus souvent considérés mutuellement comme des ennemis et qui ont eu ou se prêtent encore des prétentions territoriales l'un au détriment de l'autre. Sur l'État albanais pèse la menace d'une annexion par la Grèce de l'«Épire du Nord». Cette dénomination, officiellement inconnue dans la toponymie albanaise, recouvre dans sa version minimale les régions albanaises de Korçë et de Gjirokastër et, dans sa version maximale, tout le Sud de l'Albanie, jusqu'au fleuve Shkumbin. Le territoire ainsi défini est censé faire partie des terres grecques depuis les temps les plus reculés, et le christianisme orthodoxe y est vu comme le signe du caractère grec de la population ${ }^{2}$. À ce titre, l'Épire du Nord a été revendiquée, selon les époques, par la Grèce ou par des éléments nationalistes grecs, en particulier au sein de l'Église. Au début de la Première Guerre mondiale (1914), un éphémère «Gouvernement autonome de l'Épire du Nord» a été mis en place par des [248] éléments pro-grecs, avec pour centre la ville de Gjirokastër et pour chef un ancien ministre des affaires étrangères de Grèce, Jorgos Zographos. Après la Seconde Guerre mondiale, la revendication sur l'Épire du Nord s'est limitée à certains éléments nationalistes grecs. La question n'est redevenue d'actualité que dans les années 1990, avec l'arrivée en Grèce de nombreux Épirotes du Nord et la libéralisation des communications entre les deux côtés de la frontière, mais s'est déplacée sur le sort de la minorité grecque d'Albanie, sous la dictature comme aujourd'hui, et sur les moyens d'empêcher sa disparition par émigration ou assimilation ${ }^{3}$.

Les nationalistes albanais ont pour leur part des revendications territoriales sur la Çamëri, qu'ils considèrent comme faisant partie intégrante du territoire national, et comme ayant été injustement attribuée à la Grèce en 1913. Le terme recouvre une région allant du fleuve Pavllë au nord jusqu'à Preveza, sur le golfe d'Arta, au sud, dont seul l'extrême nord, autour du gros village de Konispol, a été attribué à l'Albanie, malgré la présence d'une importante population albanophone. Les habitants musulmans de la région ont été progressivement poussés au départ par les Grecs et beaucoup d'entre eux ont trouvé refuge en Albanie à la fin de la Seconde Guerre mondiale. Il ne semble pas y avoir jamais eu de projet d'annexion de la Çamëri par l'Albanie depuis $1913^{4}$, mais les associations de réfugiés Çams en Albanie, apparues dans les années 1990, sont assez actives et virulentes pour réclamer le retour dans leurs biens ou une compensation financière de la part de la Grèce,

\footnotetext{
2 Sur le plan religieux, la population du Sud albanais se divise en musulmans (sunnites et bektachis) et en chrétiens orthodoxes (albanophones, aroumanophones, hellénophones, slavophones). Ces derniers sont généralement évalués à $20 \%$ de la population du pays, contre $70 \%$ pour les musulmans.

3 Ainsi tout en offrant des facilités à l'immigration des Grecs d'Albanie, la Grèce s'efforce de maintenir une présence hellénophone en Albanie du Sud, par exemple en versant aux personnes âgées une pension qui leur permet de rester dans les villages de la minorité plutôt que de partir vers les villes ou l'étranger, ou encore en favorisant l'enseignement de la langue grecque.

${ }^{4}$ A l'exception de la période de la Seconde Guerre mondiale, quand l'annexion fut réalisée à la faveur de l'occupation italienne, puis allemande.
} 
ce que cette dernière refuse catégoriquement ${ }^{5}$. Alors que pendant la dictature le sujet était peu abordé, les publications se multiplient dans les années 1990 et 2000, avec un caractère souvent très revendicatif ${ }^{6}$. En 2001, des rumeurs circulaient des deux côtés de la frontière sur l'existence d'une "Armée de libération de la Çamëri "), à [249] l'image de celle qui était à la même époque responsable de la rébellion armée des Albanais de Macédoine. Ces rumeurs, que l'on crût destinées à donner une image négative des Albanais, comme celle de fanatiques prêts à enflammer les Balkans, trouvèrent un écho chez certains Albanais qui y voyaient un moyen de faire peser sur les Grecs une menace équivalente à celle que les migrants ressentent en Grèce. Mais les Çams d'Albanie qui émigrent vers la Grèce se considèrent comme les moins bien considérés des travailleurs Albanais, car les autorités les soupçonnent de menées irrédentes.

Cette frontière contestée a plusieurs fois été transgressée. Dès sa reconnaissance en 1913, elle est traversée par des irréguliers venus de Grèce (les andartes) qui exercent des pressions pour que la population se prononce en faveur du rattachement à la Grèce. Dès le début de la Première Guerre mondiale, les troupes grecques occupent Gjirokastër et Korçë, où elles font place en 1916 à une occupation italienne dans la première région, et française dans la seconde, qui dure jusqu'au début des années 1920. Pendant la Seconde Guerre mondiale, c'est depuis l'Albanie, occupée à partir du 7 avril 1939, que l'Italie se lance à l'assaut de la Grèce, en octobre 1940. Rapidement repoussées, les troupes italiennes reculent en Albanie où elles sont suivies par les Grecs qui occupent à nouveau les régions de Korçë et de Giirokastër, que le gouvernement de Métaxas comptait bien annexer, avant de reculer à leur tour, lorsque les troupes allemandes attaquent la Grèce, par la frontière bulgare et en Crète.

On comprend que dans ce contexte l'État communiste fut confronté, dès sa mise en place en 1944, à la défense de sa frontière sud. Une demande grecque déposée à la Conférence de paix de Paris, en août 1946 et rapidement retirée, proposait de rediscuter la frontière avec l'Albanie, au détriment de cette dernière ${ }^{8}$. Présent à la Conférence, Enver Hoxha réagit devant la presse : «Depuis la libération de mon pays, les monarcho-fascistes grecs ne font que nous provoquer à notre frontière sud ; ils se livrent presque chaque jour à des attaques armées contre nos gardes-frontière, contre notre peuple travailleur et épris de liberté, contre nos ports et notre littoral. (...) Je déclare solennellement que ni la Conférence de la Paix, ni la Conférence des Quatre, ni aucune autre conférence ne peuvent

\footnotetext{
5 Voir les déclarations du président grec Stephanopoulos lors de sa visite en Albanie, en octobre 2004 (http:/ /www.info-grece.com/article.php?sid=1992, consulté en décembre 2005).

${ }^{6}$ Voir par exemple Hoxha 2000 et Kondi 2001. Les revendications sur la Çamëri s'inscrivent dans le contexte général du retour du nationalisme dans le débat public en Albanie depuis la fin de la dictature ; elles ne sont donc pas limitées au mouvement nationaliste çam et s'appuient sur des revendications et des arguments déjà en vigueur avant l'époque de la dictature.

7 La même rumeur a été relancée en novembre 2003 à la suite d'un article publié dans l'hebdomadaire allemand Der Spiegel 46/2003. Voir le commentaire «L'Épire grecque, prochain théâtre d'action de la guérilla albanaise ? », publié dans Outrinski Vesnik du 11 novembre 2003, traduit sur le site du Courrier des Balkans le 13 novembre 2003 (http:/ /www.balkans.eu.org/article3795.html, consulté en décembre 2005).

${ }^{8}$ Les revendications grecques portaient également sur le Dodécanèse (jusqu'alors italien) et la frontière bulgare.
} 
remettre en discussion les frontières de notre pays en deçà desquelles il n'y a pas un pouce de terre étrangère » (Hoxha 1985) ${ }^{9}$. De fait, la guerre civile grecque, qui dure jusqu'en 1949, met non seulement la frontière gréco-albanaise en péril (elle est fréquemment traversée par les combattants), mais fait planer la menace d'une invasion et d'une [250] annexion du Sud albanais par la Grèce, tandis que cette dernière se sent elle-même menacée sur sa frontière nord (Woodhouse 2002, p. 189-190).

L'état de guerre entre les deux pays, qui a son origine dans l'agression de la Grèce par les Italiens depuis le territoire albanais (et avec une participation limitée de troupes albanaises), se maintient jusque en 1987, date à laquelle il n'est que partiellement levé ${ }^{10}$, ce qui limite fortement les communications transfrontalières, que ce soit au niveau des États ou à celui des communautés locales. Les rares personnes qui traversent le font en général pour quitter clandestinement l'Albanie ou pour y mener des opérations secrètes.

\subsection{Hétérogénéité culturelle. Les risques de la périphérie et du "transnationalisme »}

Le compromis atteint sur le tracé de la frontière a pour conséquence d'une part de laisser insatisfaites les revendications territoriales des deux États voisins, donnant ainsi lieu à l'idée d'un espace national à rattacher au corps de l'État, d'autre part de laisser de chaque côté des «minorités nationales", dont la loyauté envers l'État est rapidement mise en doute.

Du coté albanais, un groupe d'hellénophones assez compact occupe les régions du Dropull et du Pogon, dans le district de Gjirokastër, celle du Vurg dans le sud des districts de Delvinë et Sarandë et quelques villages de la vallée de la Vjosë (district de Përmet). Selon les estimations, et surtout en fonction de la définition qui est retenue, la taille de ce groupe varie de 60000 (recensement albanais de 1989) à 400000 personnes (nationalistes grecs). Dans le premier cas, ne sont pris en compte que les habitants de "nationalité ${ }^{11}$ grecque des 99 villages constituant la minorité grecque d'Albanie; dans le second, les chiffres incluent l'ensemble des chrétiens orthodoxes d'Albanie ${ }^{12}$. Une minorité macédonienne (slavophone) plus réduite (4 700 personnes en 1989 d'après le dernier recensement général de la population albanaise à tenir en compte ce critère, entre 60000 et 100000 selon les

\footnotetext{
9 Si la Grèce n'obtient satisfaction ni en Albanie, ni en Bulgarie, c'est que ces deux pays sont alors protégés par l’URSS. Voir Chiclet 2000, p. 228.

10 Les dispositions interdisant la restitution ou l'indemnisation des biens des albanophones de Grèce aujourd'hui citoyens albanais n'ont pas été supprimées. Ce point fait encore l'objet d'un contentieux entre les deux États (voir http://www.balkans.eu.org/article4388.html, consulté en décembre 2005).

11 Il s'agit de la kombësi. Sur le modèle soviétique et yougoslave, l'État albanais distingue la «citoyenneté » (nënshtetësi) de la «nationalité » (kombësi) de ses ressortissants.

${ }^{12}$ Les chiffres maxima procèdent de l'hypothèse radicale que l'ensemble des chrétiens orthodoxes d'Albanie seraient des Grecs albanisés de force sous Enver Hoxha. Pour les débats concernant l'importance de la minorité grecque, voir Zarrilli 1999, p. 73. L'hypothèse inverse (les Grecs d'Albanie sont des Albanais hellénisés) existe également.
} 
sources macédoniennes) habite à proximité de la frontière gréco-macédonienne (ARYM), dans le district de Korçë $\ddot{~}^{13}$.

[251] La minorité grecque est perçue comme une menace à plusieurs titres. Sa seule présence sur le territoire national pose d'abord la question du caractère proprement « national» de cet espace et des frontières qu'il convient de lui reconnaitre. Elle semble en effet justifier, au moins en partie, les prétentions grecques sur l'Épire du Nord, qui remontent à l'époque de la création de la frontière. Elle est ensuite suspectée de loyauté envers l'État grec et de déloyauté envers l'État albanais, ce qui la met dans la position d'un ennemi de l'intérieur. Enfin, elle est censée servir de relais à la politique d'hellénisation des Albanais chrétiens orthodoxes, que l'État albanais attribue à la Grèce.

Du coté grec, des groupes albanophones - musulmans et chrétiens - occupent à l'époque de l'établissement de la frontière une partie de la Thesprotie et de la Macédoine occidentale, à l'ouest de Kastoria. Ceux de Thesprotie sont appelés Çams en albanais et Tsamidès en grec, et leur région est désignée sous le nom de Çamëri/Tsamouria. Sur décision de la commission mixte du 19 janvier 1923, les musulmans ont été exclus de l'échange de population avec la Turquie en 1924 et ont pu rester sur place ${ }^{14}$. Ils ont toutefois progressivement quitté la Grèce pour l'Albanie et d'autres pays (Turquie, ÉtatsUnis) dans les années 1920 et 1930, puis brutalement à la fin de la Seconde Guerre mondiale, lorsqu'ils furent accusés de collaboration avec les occupants italiens et allemands et durent renoncer à des biens fonciers qui avaient déjà été réduits par la réforme agraire mise en œuvre par la Grèce entre 1913 et 1924, mais que les occupants leur avaient rendus (Sivignon 1989, p. 58-59). Une estimation récente évalue leur nombre de 23000 à 25000 dans les années 1940, contre 127 au recensement grec de 1951 (Kretsi 2002, p. 173 et 186). Les musulmans de Macédoine occidentale en revanche, n'ont pas été exclus de l'échange de population et ont dû partir en Turquie en 1924; un certain nombre d'entre eux ont toutefois préféré traverser illégalement la frontière et s'installer en Albanie (de Rapper 1997). Dans les deux cas, les chrétiens albanophones se sont assimilés à l'identité grecque dominante au point de ne jamais former de minorité.

En dehors de ces «minorités nationales ${ }^{15}$ ", deux groupes transfrontaliers ne s'identifient à aucun des deux États et n'ont que récemment été considérés, du côté albanais, en tant que "minorités linguistiques et culturelles». Il s'agit des Tsiganes - que l'on rencontre sur l'ensemble du territoire des deux pays - et des Aroumains, pasteurs de langue romane que [252] la vie semi-nomade menait des estives montagnardes aux hivernages côtiers et que l'on trouve sédentarisés aujourd'hui essentiellement dans les villes

\footnotetext{
13 Sur les minorités en Albanie voir Beci 1998 (version officielle) et surtout Barjaba 1995. Sur la minorité grecque en particulier, Tsitselikis, Christopoulos 2003.

14 Il s'agit de l'échange appliqué à la suite du traité de Lausanne de 1923, qui sanctionnait la défaite militaire grecque de 1922 en Asie mineure, face à la Turquie : les citoyens musulmans de Grèce, à l'exception de ceux de Thrace, sont envoyés en Turquie, tandis que les citoyens de religion grecque orthodoxe de Turquie, à l'exception de ceux d'Istanbul et des îles d'Imbros et Ténédos, sont envoyés en Grèce.

15 On trouve enfin des Slavophones dans la partie septentrionale de la Macédoine grecque, mais ils ne concernent pas directement notre frontière.
} 
de Vlorë, Fier, Korçë, Gjirokastër, Sarandë, Kastoria et Ioannina, ainsi que dans un grand nombre de villages de ces régions. Ils étaient 4220 à se déclarer en tant que tels au recensement albanais de 1955 et 10000 au recensement de 1959 (Barjaba 1995, 95), ce qui illustre les variations que connaît l'identification à la catégorie "Valaque » lors des recensements. On sait par ailleurs qu'il existe une tendance à la sous-déclaration des populations aroumaines dans les autres pays balkaniques (Gossiaux 2002, p. 172). La catégorie disparaît du recensement de $1989^{16}$. Selon les associations aroumaines, jusqu'à 250000 Aroumains vivraient aujourd'hui en Albanie, tandis que les travaux les plus récents les estiment à 100000 personnes (Kahl 2002, p. 156). En Grèce, ils étaient 22736 à se déclarer en tant que tels en 1951 lors du dernier recensement à prendre en compte cette catégorie linguistique. Les nationalistes aroumains avancent le nombre de 600 000, mais d'autres estiment qu'ils ne dépassent pas les 300000 (Kahl 2002, p. 153) ${ }^{17}$.

Les Tsiganes ne sont pas perçus comme un risque pour l'État, dans la mesure où ils ne sont soutenus par aucun pays voisin et n'ont pas de revendications politiques susceptibles de mettre la frontière en péril. Par leurs activités commerciales qui les mènent à s'approvisionner en Grèce et par leur apparente facilité à traverser la frontière, ils participent néanmoins à l’image de la zone frontière comme zone de danger, parce que mal fréquentée.

Le statut des Aroumains en Albanie présente également une ambiguïté. Leur mode de vie traditionnellement nomade les associe à l'espace sauvage de la montagne et suscite la suspicion parmi les populations villageoises. Même sédentarisés et employés dans les coopératives agricoles, comme ce fut le cas dans les régions de Lunxhëri et du Pogon à partir de la fin des années 1950, ils forment une communauté à part, avec laquelle on ne se marie pas. Le régime communiste considérait pourtant qu'ils ne formaient pas une minorité à part entière et que leur petit nombre, comme leur assimilation poussée à la population albanaise (favorisée par l'absence d'enseignement primaire dans leur langue), justifiait la suppression de la catégorie "Valaque » dans les recensements comme dans les documents d'identité. Dans le district de Gïrokastër, où les Aroumains sont originaires du village de Kephalovrisso, situé dans le Pogon grec, ils semblent avoir été [253] généralement suspectés de sympathie pour les Grecs de la part des autorités locales, et ont subi à ce titre une certaine forme de persécution. On les accusait de servir de relais aux espions et saboteurs que l'on disait envoyés en Albanie depuis l'autre côté de la frontière.

La présence de ces minorités est perçue comme un risque de déstabilisation par l'État albanais. Avec l'instauration du multipartisme en décembre 1990, des organisations et associations minoritaires ont apparu. La Parlement albanais a cependant voté une loi interdisant les partis politiques à base ethnique (ou religieuse), qui a pour conséquence que

\footnotetext{
16 Malgré cette disparition, la rubrique «autre nationalité » du formulaire de recensement a recueilli 782 déclarations «Valaques » sur 64816 non Albanais : 400 dans le district de Gjirokastër, environ 150 dans ceux de Korçë et de Sarandë.

${ }^{17}$ En comptant tous les gens qui se disent «Armâní, Rrămăńi or Vlaşi (Aromanian) » et qui comprennent la langue. Les seuls locuteurs de l'aroumain serraient 100000 en Grèce (Kahl 2002, p. 153). Il ne faut pas perdre de vue que ces estimations portent sur une catégorie extrêmement fluide.
} 
les minorités ne sont pas directement représentées dans le système politique. Le Parti des droits de l'homme - officiellement sans base ethnique - est cependant généralement considéré comme le représentant des minorités et de la minorité grecque en particulier. Plus récemment, les réactions violentes suscitées dans le monde politique et intellectuel par la publication en 2003 du nouvel atlas de la population albanaise, fruit d'une coopération germano-albanaise, illustrent l'extrême sensibilité de la question des minorités en Albanie. Les auteurs de l'atlas évaluent en effet à 10,8\% la proportion de population non albanaise, tandis que le recensement de 2001 n'en reconnaît que 2\%. La différence tient à la reconnaissance par l'atlas des Tsiganes et des Aroumains en tant que "minorités ethniques » alors que la classification officielle ne les reconnait qu'en tant que " minorités culturelles », ce qui ne justifie pas leur catégorisation parmi la population non albanaise ${ }^{18}$.

Par rapport au reste du pays, et du fait de l'hétérogénéité culturelle dont nous venons de parler, les régions frontières présentent ainsi la double particularité d'être différentes par le peuplement et les modes de vie, et d'avoir plus de choses en commun avec l'autre côté de la frontière. Cela est vrai pour les Aroumains du district de Gjirokastër qui pour nombre d'entre eux sont originaires du village grec de Kephalovrisso, et se sentent très proches des Aroumains de Grèce, et suffisamment éloignés de leurs voisins albanais pour maintenir une endogamie de communauté. C'est le cas bien évidemment de la minorité grecque d'Albanie qui entretenait avant l'établissement et la fermeture de la frontière des liens très étroits avec l'autre côté. L'exemple le plus flagrant est celui du Pogon, dont une partie a été attribuée à l'Albanie et l'autre à la Grèce (Green 2005). Ces exemples les plus évidents ne doivent pas faire oublier cependant que certains Albanais du Sud, sans pour autant revendiquer une origine grecque, estiment avoir beaucoup de choses en commun avec la Grèce et se distinguer en cela du reste des Albanais (de Rapper 2004). Parmi les chrétiens orthodoxes albanophones, le [254] risque - ou la tentation - de l'hellénisation semble avoir été suffisamment fort pour que l'on attribue localement à l'État des mesures visant à la contrer. Plusieurs informateurs chrétiens de Lunxhëri nous ont ainsi raconté que la création par le régime communiste d'un certain nombre de villages dont la population avait été amenée de régions musulmanes avait pour but d'établir une sorte de cordon sanitaire entre leur région et celle de la minorité grecque, afin d'empêcher l'hellénisation de la première au contact de la seconde (de Rapper 2005). Dans la même région, on peut également entendre que, sans la proximité et l'aide de la Grèce, les chrétiens auraient été convertis à l'islam, comme le furent les habitants de la région voisine et aujourd'hui musulmane du Kurvelesh ${ }^{19}$. D'un côté, les communistes empêchent des Albanais de devenir grecs, de l'autre, les Grecs empêchent les chrétiens albanais de devenir musulmans. Ces spéculations concernant le rôle des uns et des autres dans le paysage identitaire actuel semblent révélatrices des incertitudes et de l'instabilité des appartenances qui résultent de la conjonction de la frontière internationale et des principes de division en vigueur dans la société locale de cette région. En même temps, l'importance que revêt pour l'État-nation la frontière

\footnotetext{
${ }_{18}$ Voir l'article publié par la revue Klan en décembre 2003 (traduction française sur le site du Courrier des Balkans, http:/ /www.balkans.eu.org/article3938.html, consulté en décembre 2005).

${ }^{19} \mathrm{La}$ mémoire collective garde le souvenir de conversions forcées à l'époque d'Ali pacha de Ioannina, au début du XIX ${ }^{\mathrm{e}}$ siècle.
} 
internationale exige des populations locales des allégeances nettes et rend suspecte toute position ambiguë quant à l'appartenance nationale.

\section{Implications. Construction et nationalisation de la frontière}

En dehors du risque du conflit international, la frontière fait ainsi apparaitre une autre forme de risque dans les relations que les populations frontalières entretiennent avec les États ou, si l'on veut, la périphérie avec le centre. Ces deux manifestations de la notion de risque suscitent trois types de réponses de la part de l'État albanais et des populations frontalières. La première est militaire et consiste à imposer un contrôle étatique sur la région frontière et sur les traversées de la frontière ; la deuxième est idéologique : il s'agit de renforcer le sentiment national des populations frontalières et de donner une signification forte à la frontière ; la troisième est statistique et consiste à canaliser les appartenances aux minorités nationales afin de favoriser leur assimilation. L'intensité de ces réponses a diminué au début des années 1990, comme a diminué, d'une manière générale, l'efficacité de l'action de l'État albanais, mais leurs effets sont toutefois toujours présents.

\subsection{La fortification et la militarisation de la frontière}

[255] Dès les années d'après-guerre, les frontières deviennent le lieu d'une menace pour l'État albanais qui se met en place sous l'autorité d'un Parti communiste tout-puissant. Le régime redoute l'infiltration d'agents étrangers et la guerre civile grecque, qui s'achève en 1949 par des opérations sur un terrain très proche de la frontière albanaise, renforce le sentiment d'une menace militaire pesant sur la frontière.

Dans les années 1960, après la rupture de l'Albanie avec l'Union soviétique, la défense du pays ne peut plus reposer sur une aide militaire extérieure. Sous la pression de la Chine, nouvel mais lointain allié, la défense du pays répond désormais à une nécessité d'autonomie. Au début des années 1980, après sa rupture avec la Chine en 1978, l'Albanie se retrouve isolée, et lance un vaste programme de construction de fortifications. Il s'agit de multiplier les lignes de défense parallèlement à la frontière, par d'innombrables casemates et tranchées qui marquent encore le paysage frontalier albanais (Jandot 1994, p. 181-182). Dans le même temps, une clôture suit la ligne frontière jusqu'à un kilomètre à l'intérieur du pays. C'est le klon: haut de trois mètres, fait de fils de fer barbelés munis de grelots ou légèrement électrifiés pour faire retentir une sonnerie en cas de passage forcé, il parcourt toute la zone frontière, y compris les endroits les plus inaccessibles. De chaque côté, sur une largeur de quatre mètres, le sol est soigneusement débroussaillé, nettoyé et ratissé, avec parfois un apport de sable. Cette «bande molle» (brez i butè), régulièrement inspectée par les patrouilles, doit garder l'empreinte de tout ce qui s'en approche. Entre le klon et la ligne frontière proprement dite s'étend la « zone interdite " (zonë e ndaluar) où toute personne non autorisée est abattue sans sommation. Par endroit, des "portes » (porta) s'ouvrent dans le klon, à proximité d'un poste de garde. Elles permettent l'accès des gardes-frontières à la zone interdite et sont également utilisées, le cas échéant, par les brigades des coopératives 
agricoles qui vont travailler les terres de la zone interdite. Celle-ci est en général inhabitée (seuls de rares villages, comme celui de Vidohovë dans le Devoll, se trouvent pris entre la ligne frontière et le klon), mais les impératifs de rendement agricole font que, dans les vallées et les régions peu accidentées, on y envoie des ouvriers agricoles (sous la surveillance de l'armée, et choisis parmi les plus fidèles au régime) et des bergers.

Le klon, présenté d'abord comme un rempart contre les infiltrations depuis l'extérieur, devient vite un moyen d'empêcher le départ des Albanais vers l'extérieur. La région est d'ailleurs soumise à un contrôle des déplacements beaucoup plus sévère et les villages les plus proches de la frontière ne sont accessibles qu'avec une autorisation. Les populations sont elles-mêmes mises à contribution et encouragées à signaler ou dénoncer tout comportement [256] suspect à proximité de la frontière. Des récits rapportant l'arrestation de fuyards potentiels par des villageois vigilants attestent que l'objectif fut en partie atteint ${ }^{20}$. On entend en revanche peu de récits racontant comment des villageois sont venus en aide aux fuyards : un tel comportement était extrêmement risqué. Les fuyards étaient en effet considérés comme des «traîtres à la patrie » et passibles de la peine capitale, tandis que leur famille restée dans le pays subissait l'emprisonnement ou l'internement en camp de travail. L'exil intérieur (internim) dans une région éloignée de la frontière était de même infligé aux complices locaux, l'État ne tenant pas à garder des populations peu sûres à proximité de la frontière.

Le klon est démantelé au printemps 1991. Il disparaît complètement, mais la «bande molle » reste visible comme une cicatrice après plus de dix ans. Les postes de garde sont abandonnés, certains sont démontés par les villageois qui récupèrent les matériaux de construction. De la même manière, le fil de fer barbelé est réutilisé pour clôturer les nouvelles propriétés privées issues de la décollectivisation ${ }^{21}$.

Dans les années 1990, la démilitarisation de la ligne frontière s'accompagne du développement des points de passage et de la mise en place d'un contrôle douanier, non seulement au niveau des postes frontière, mais aussi dans les villages susceptibles de servir de relais à la contrebande. La menace n'est plus perçue comme une menace militaire, mais comme pesant sur le commerce légal et les gains qu'il procure à l'État. L'incident d'avril 1994, dans le village de Peshkëpi, montre cependant que la frontière reste un lieu sensible dans lequel l'État peut être attaqué depuis l'extérieur ${ }^{22}$.

Du côté grec, la menace militaire et le risque d'invasion armée ont disparu, pour être toutefois remplacés par une autre invasion, celle représentée par l'immigration albanaise. La

\footnotetext{
${ }^{20}$ Vasil Konomi raconte l'histoire d'un fuyard trompé et dénoncé par les habitants d'un village de la minorité grecque, alors qu'il pensait avoir déjà atteint la Grèce, puisqu’il entendait parler grec (Konomi 2001, p. 95-98). Des histoires de dénonciations sont également entendues dans le Devoll.

21 Loi de juillet 1991 sur le partage des terres agricoles, précédée par une mesure qui rétablit le lopin individuel.

${ }^{22}$ En avril 1994, un poste de garde albanais situé dans le village de Peshkëpi (Gjirokastër) est attaqué par des inconnus venus de l'autre côté de la frontière. L'attaque est revendiquée par le «Front de libération de l’Épire du Nord». Voir Le Monde du 14 avril 1994.
} 
présence militaire et policière reste visible, sous la forme de contrôle sur les routes, de patrouilles, de postes d'observation ou encore de tranchées coupant les chemins transfrontaliers pour en interdire le passage aux voitures. La frontière reste, du fait de cette nouvelle conjoncture, le lieu d'une prise de risque pour ceux qui cherchent à la traverser.

\subsection{L'établissement d'un paysage idéologique conforme}

[257] La frontière est un lieu à défendre. Dans le langage de l'État communiste, la sécurité n'y est jamais acquise, en raison des "provocations » (provokim) des ennemis, qui cherchent à mettre en péril l'existence de l'État albanais. Chaque incident, chaque opération militaire est une provocation. C'est le cas de la «provocation navale » du 22 octobre 1946, lorsqu'un navire britannique heurte dans le détroit de Corfou, dans les eaux territoriales albanaises, des mines laissées par les Allemands pendant la guerre. L'Albanie refuse de compenser l'Angleterre pour les dégâts occasionnés et l'accuse de provocations. Londres répond en confiscant l'or de la Banque nationale de Tirana, que les Allemands avaient envoyé en Allemagne à la fin de la guerre. "Tout cela, écrit Amik Kasoruho en 1991, apportait de l'eau au moulin de la propagande qui présentait l'Albanie comme étant au centre des objectifs impérialistes anglo-américains et justifiait les mesures prises par le gouvernement de [Enver] Hoxha, y compris la fermeture des missions diplomatiques américaine et anglaise à Tirana » (Kasoruho 1996, p. 53).

Les auteurs de ces provocations, selon les Albanais, sont le plus souvent les Grecs. «Pendant les années 1946 et 1947, écrit Mentar Belegu en 1968, les monarcho-fascistes grecs, tout en clamant dans les conférences internationales que l'Albanie était en état de guerre avec la Grèce, et que Korçë et Gjiroksatër étaient des "terres grecques”, ont violé des centaines de fois les frontières, les eaux territoriales et l'espace aérien de notre patrie » (Belegu 1968, p. 196-197). Les dernières opérations de la guerre civile grecque, dans les montagnes du Grammos et du Vitsi, se déroulent en vérité à proximité de la frontière albanaise ; elles sont présentées comme des attaques visant l'Albanie et le Parti communiste au pouvoir qu'elles cherchent à déstabiliser (Madhi 1967, p. 44). C'est d'ailleurs suite aux derniers combats, en août 1949, au cours desquels intervient une compagnie de l'armée albanaise, que la frontière est fermée par Enver Hoxha (Chiclet 2000, p. 229). Elle le restera jusqu'en janvier 1985, tandis que, dans la propagande comme au sein des populations frontalières, la frontière devient le lieu d'une défense héroïque de la patrie. Au début des années 1950, alors que la collectivisation des terres agricoles ${ }^{23}$ rencontre des résistances, les voisins yougoslaves et grecs sont accusés d'envoyer en Albanie des «bandes de d'agitateurs » (diversant) qui se lient avec l' " ennemi de classe intérieur », c'est-à-dire avec les cultivateurs qui refusent de rejoindre les coopératives agricoles et sont pour cette raison classés dans la catégorie des kulak (Madhi 1967, p. 46).

${ }^{23}$ Les collectivisations débutent dès 1946 mais leurs conditions sont durcies à partir de décembre 1955. En 1958, elles concernent $63 \%$ des surfaces du pays (Daniel 1996, p. 136). 
[258] La mise en place du paysage idéologique de la frontière s'accompagne d'une préparation militaire et psychologique de la population. Pour des raisons de sécurité, les gardes-frontières n'étaient pas recrutés sur place, mais dans des régions lointaines. Cela relève des "risques du transnationalisme» et de la suspicion à l'égard des populations frontalières, que leur bonne connaissance du terrain et leurs liens avec l'autre côté pouvaient aider à traverser. En conséquence, la méfiance régnait entre la population et ceux qui étaient chargés tout autant de défendre la frontière depuis l'extérieur que d'empêcher les évasions, et les gardes-frontières pâtissaient d'une mauvaise réputation; on les taxait facilement d'ignorance et de brutalité. En plus de cela, la population était soumise à des périodes d'entraînement militaire (les zbor) et à des alertes impromptues. Les habitants du Devoll se souviennent de ces exercices qui les menaient à l'assaut des montagnes pour stopper les "capitalistes et les révisionnistes »: «Les exercices avaient lieu aussi pendant la nuit, raconte l'un d'eux, et à l'approche des jours fériés, car c'est le moment que choisissait l'ennemi pour envahir le pays. Alors, les jours de vacances, on les passait le fusil à la main. ».

Si la chute de la dictature et l'ouverture de la frontière en 1991 ont modifié le paysage frontalier, elles ne le transformèrent pas totalement. L'ouverture a d'abord une dimension idéologique dans la mesure où il est désormais permis de parler de la frontière, ce qui était interdit à l'époque de la fermeture, de même que de pointer le doigt vers la frontière. C'est aussi la remise en cause d'un discours unique sur la frontière : celle-ci n'est plus seulement le lieu d'une menace, un rempart extérieur qu'il faut défendre et surveiller. Les frontaliers deviennent les habitants d'une zone aux particularités indiscutables dont on réinvente le passé commun à travers les récits sur la fermeture et surtout sur l'avant fermeture, à l'époque où des liens familiaux et commerciaux unissaient les deux côtés.

Pourtant, la réconciliation n'est pas la règle générale. Les premiers "réfugiés » albanais furent certes bien accueillis et aidés par la population des villages grecs, mais l'accroissement du nombre de migrants et les mesures officielles prises par la Grèce pour limiter l'entrée illégale sur son territoire ont empêché qu'un climat de confiance s'établisse entre les deux populations. D'une certaine manière, la figure du Grec chez les Albanais de la frontière est toujours celle d'un ennemi. Les Grecs apparaissent comme des ingrats dans leur façon de traiter les migrants albanais. Ceux-ci ne sont-ils pas en train de reconstruire la Grèce ? Les Grecs n'ont-ils pas été accueillis par les Albanais pendant la Seconde Guerre mondiale, quand la famine sévissait en Grèce alors que l'Albanie bénéficiait d'une meilleure situation? Les récits à propos des violences dont sont victimes les clandestins et les articles de journaux rapportant les cas de nombreux réfugiés qui trouvent la mort en traversant la frontière rappellent que cette dernière, même si le dispositif de l'État autoritaire a disparu, peut encore être mortelle, et demeure un obstacle [259] objectif à la libre circulation des personnes. Enfin, par leur politique à l'égard des migrants, qui se sentent obligés de se déclarer chrétiens et d'adopter des prénoms grecs, les Grecs sont accusés de vouloir helléniser l'Albanie du Sud.

La réconciliation n'a pas beaucoup plus réussi chez ceux qui avaient le sentiment d'avoir été injustement séparés des leurs par la frontière. Les enquêtes montrent ainsi que la 
solidarité entre les Aroumains des deux côtés de la frontière n'a pas été totale et que la confiance et la compréhension n'ont pas toujours été la règle (Sintès 2003). De telles "déceptions» se rencontrent chez les membres de la minorité qui sont partis vivre en Grèce.

L'ouverture des frontières de l'Albanie ne signifie donc pas la fin du risque pour ceux qui la traversent.

\subsection{La mise en place de la minorité grecque}

La politique de l'Albanie à l'égard de la minorité grecque a une grande influence sur le devenir de la région frontière ${ }^{24}$. Dès la reconnaissance officielle de la minorité par le gouvernement albanais, en 1921, celle-ci se trouve définie sur une base territoriale tout autant que linguistique ou culturelle. Les droits minoritaires ne s'appliquent en effet qu'aux Grecs vivant dans les régions de Gjirokastër et de Sarandë, tandis que les trois localités de Himarë, Dhërmi et Palasë, sur la côte de la mer ionienne, se voient reconnaittre le droit à l'enseignement en grec, même s'ils ne sont pas reconnus comme appartenant à la «minorité dans le plein sens du terme $e^{25} »$. Les Albanais refusent de reconnaître une présence grecque dans la région de Korçë pourtant revendiquée par Athènes. En 1945, après la prise de pouvoir par les communistes, la zone grecque est réduite des trois derniers villages, pour se limiter aux 99 villages de Gjirokastër et Sarandë (Kondis, Manda 1994, p. 16 et 21). On raconte que les villages de la minorité qui s'étaient battus du mauvais côté pendant la guerre furent privés de leur statut de minorité et albanisés, du simple fait que la langue grecque y devenait dès lors interdite, de même que ceux qui avaient " mal voté » lors des élections de 1946 (Tsitselikis, Christopoulos 2003, p. 29). La limitation numérique de la minorité se faisait également en incitant les gens qui quittaient le territoire de la minorité pour des raisons professionnelles ou pour se marier à ne pas déclarer leurs enfants comme étant de nationalité grecque. "Il ne fallait pas réclamer la nationalité grecque pour ses enfants, écrit Vasil Konomi à propos des Grecs qui partaient en ville, car de cette manière on était classé comme quelqu'un qui soutenait l'ennemi » (Konomi 2001, p. 87).

[260] L'histoire et l'ethnologie sont mises à contribution pour minimiser l'importance de la présence grecque en Albanie. Dès l'entre-deux-guerres, la région de Himarë avait été déclarée albanaise et ses habitants n'étaient reconnus en tant qu'hellénophones qu'en raison des liens commerciaux qu'ils entretenaient depuis longtemps avec Corfou. Quant aux Grecs du Dropull, on prétend qu'ils sont d'installation récente dans leurs villages, où ils sont venus, depuis la Grèce du Sud, pour travailler sur les terres des propriétaires albanais (musulmans), au temps d'Ali pacha de Ioannina.

Contrairement à ce que l'on entend souvent en Grèce, les Grecs de la minorité n'ont pas le sentiment d'avoir été persécutés en tant que tels. «Dans les années de la dictature

\footnotetext{
${ }^{24}$ Sur la mise en place de la minorité grecque dans l'entre-deux-guerres, voir Clayer 2004.

${ }^{25}$ Selon une formule employée par le ministre de l'Éducation et citée dans Clayer 2004, p. 171.
} 
communiste, écrit Vasil Konomi, originaire du Pogon, [la minorité] a été traitée indistinctement, de la même manière que tout le peuple albanais. Si les Albanais, pendant presque cinq décennies, ont subi les prisons, les internements politiques, la pauvreté et l'isolement, la minorité grecque les a subis également» (Konomi 2001, p. 85). Un informateur du Dropull vivant à Gjirokastër explique que les Grecs ne se sentaient pas déconsidérés par rapport au reste de la population, car « la dictature n'encourageait pas la haine ethnique ». Le Dropull, selon lui, était « aidé par le régime plus que les autres régions, pour des raisons politiques ». C'est d'ailleurs la réputation de la minorité dans le reste de la population : favorisés par Enver Hoxha qui ne voulait pas donner d'arguments à la Grèce concernant le traitement de la minorité, les Grecs sont perçus comme ayant bénéficié plus que d'autres d'améliorations de leurs conditions de vie et de l'accès aux études supérieures et aux carrières administratives ${ }^{26}$. On ne peut nier cependant que le régime menait une politique d'albanisation de la minorité, avec un certain succès, dans la mesure où un certain nombre de minoritaires ont choisi de se déclarer Albanais, "par intérêt, pour être bien avec le régime, ou par complexe», explique le même informateur. A quoi il faut ajouter la multiplication des mariages entre Grecs et Albanais, due aux encouragements du régime comme à la fermeture du marché matrimonial transfrontalier, et qui favorisaient le passage de la frontière ethnique $e^{27}$. En tant que région frontalière, la région de la minorité était par ailleurs soumise de manière intense à la "propagande rouge » à l'occasion de fréquentes visites de dirigeants du parti (Konomi 2001, p. 85).

\section{Les réponses}

[261] Le marquage physique et idéologique de la région frontière par l'État fournit un cadre à la pratique de la frontière par les populations locales. En même temps qu'elle marque un différentiel politique et économique entre les deux pays, induit par leurs systèmes politiques respectifs, la frontière s'impose à la fois comme obstacle et comme ressource. C'est ce qui apparait dans les usages que font de la frontière les communautés frontalières, en matière de commerce comme d'émigration. La typologie des usages met par ailleurs en évidence un second type de réponse aux risques de la frontière: il s'agit de l'adaptation des discours identitaires et des catégories d'appartenance aux contraintes et aux opportunités tour à tour imposées et offertes par la frontière selon les fluctuations de son statut. Tout en étant l'expression d'un certain état des frontières ethniques dans la région, la frontière internationale a un impact sur celles-ci. Le caractère mouvant et contextuel des frontières ethniques contribue à son tour à soumettre la frontière internationale à un certain nombre de pressions exercées par les différents groupes de population qui se créent à son contact.

\footnotetext{
${ }^{26}$ Les mêmes arguments sont avancés à propos de la minorité macédonienne, dans le Devoll.

${ }^{27}$ Dans le sens Grec $\rightarrow$ Albanais jusqu'en 1991, dans le sens Albanais $\rightarrow$ Grec depuis le développement de l'émigration vers la Grèce, facilitée pour les membres de la minorité.
} 


\subsection{Traverser ou tourner le dos à la frontière}

Les départs de l'Albanie vers la Grèce semblent avoir touché la minorité grecque ainsi que les Albanais chrétiens à la fin de la Seconde Guerre mondiale, lors de la prise de pouvoir des communistes. Ils concernent alors des gens qui craignent pour leur sécurité ou leurs biens dans le contexte d' "épuration» qui règne à cette époque, et qui savent le plus souvent pouvoir compter sur un réseau, familial ou autre, du côté grec de la frontière. La meilleure surveillance, puis la construction du klon, rendent ces départs de plus en plus difficiles. La plupart des habitants de la région frontalière vivent alors le dos tourné à la frontière : il ne leur est permis ni de la traverser, ni de s'en approcher, ni même d'en parler. La connaissance de l'autre côté est minimale et marquée de l'empreinte du "paysage idéologique » dont nous avons parlé plus haut. "Le vent du sud est appelé kosturare, du nom de Kostur [Kastoria], explique une habitante de la petite ville frontalière de Bilisht (Devoll). Pendant la fermeture de la frontière, il était déjà appelé comme ça, même si on ne savait pas très bien ce qu'était Kostur et où ça se trouvait, sinon que ça avait été un village albanais, aujourd'hui en Grèce ». L'interdiction de parler la langue grecque - en dehors du territoire de la minorité grecque - et de suivre les émissions radiotélévisées en provenance de Grèce renforçait cet éloignement et cette méconnaissance. L’interdit qui pesait sur la frontière encourageait le silence chez les anciens émigrants qui l'avaient souvent traversée et qui connaissait bien les villages grecs les plus proches.

[262] Malgré tout, certains tentaient la traversée. Il est difficile de connaître aujourd'hui les motivations qui les poussaient à prendre ce risque, en dehors de l'envie de s'échapper d'un régime autoritaire et répressif, et de fuir la pauvreté. Ils étaient appelés « fuyards » (të arratisurit) dans la terminologie officielle et risquaient non seulement leur vie, mais aussi celle de leur famille élargie, qui pouvait être emprisonnée ou internée, voire même celle des gardes-frontières qui les avaient laissés passer ${ }^{28}$.

La situation de fermeture de la frontière s'interrompt au début des années $1990^{29}$. Le régime s'est d'abord effondré par son centre avec la crise des ambassades en juillet 1990 à Tirana qui a vu des milliers d'Albanais forcer les grilles des ambassades étrangères pour demander des visas. Les zones frontalières ne sont pas touchées les premières par cette ouverture car elles sont étroitement surveillées mais elles sont rapidement affectées par le passage de milliers de personnes vers la Grèce et la disparition du klon (de Rapper, et al. 2000 , p. 209-210). Cette ouverture de la frontière albanaise ne signifie cependant pas que tous les obstacles ont disparu. Par l'instauration de contrôles stricts des points de passage et de la zone frontalière en général, et par la mise en place d'une législation restrictive au

\footnotetext{
28 Pour un traitement littéraire, voir Kadaré 2002.

${ }^{29} \mathrm{La}$ réouverture officielle de la frontière, en janvier 1985, se traduit par une augmentation des échanges commerciaux et par des visites de «touristes » dans les régions du Sud (souvent liés par leurs origines à la minorité grecque). En raison toutefois du maintien d'un régime qui refuse à la population tout contact avec l'étranger (l'ouverture ne signifie pas encore que les Albanais peuvent sortir librement du pays), l'impact de cette ouverture sur les régions frontalières est très faible.
} 
séjour en Grèce pour les dizaines (puis centaines) de milliers de travailleurs albanais, l'espace grec se ferme.

On assiste alors à l'émergence de nouveaux acteurs de la frontière aux échelles régionales et surtout à l'échelle des communautés locales. Commerce et émigration en sont les manifestations principales. La frontière devient ressource, elle oriente certaines activités. Ce sont d'abord les activités liées à l'émigration, qui prend des formes différentes (permanente, saisonnière, journalière), et qui s'accompagne d'activités connexes dans les villes et villages frontaliers: hébergement et approvisionnement des candidats à l'émigration, transports collectifs dédiés, guides et passeurs travaillant souvent en collaboration avec des associés grecs. Quelles que soient sa forme et ses modalités, l'émigration est une entreprise risquée. La traversée de la frontière elle-même, qu'elle se fasse légalement, par le poste frontière, ou illégalement, par la montagne, expose les migrants à une série de risques, qui vont du refoulement par la police albanaise ou grecque jusqu'à la mort dans les montagnes enneigées du Pinde septentrional, en passant par les vexations et humiliations, l'expulsion et les violences policières ${ }^{30}$. L'expérience migratoire présente par ailleurs un certain nombre de [263] risques, du fait des conditions de travail éprouvantes, de son caractère souvent clandestin qui met les migrants à la merci de leurs employeurs et des contrôles de police, ou encore des exactions de bandes criminelles qui volent ou rackettent les migrants Elle met de plus les migrants dans une position instable en leur imposant des adaptations personnelles parfois douloureuses (changement de prénom, exposition à la mauvaise réputation des Albanais) (de Rapper 2002b, p. 202-207).

Les activités commerciales se déclinent également à plusieurs échelles, depuis la contrebande de sucre ou de paille entre villages frontaliers jusqu'aux entreprises les plus florissantes dont l'activité couvre tout le pays. Elles comportent elles aussi des risques, qu'il s'agisse de ceux inhérents à la contrebande, des problèmes d'approvisionnement ou encore de la méfiance qui règne entre partenaires grecs et albanais. La création de la chambre de commerce de Gjirokastër en 1987 et surtout, son affranchissement du contrôle de l'État depuis 1992 sont la manifestation du développement des activités commerciales dans la zone frontalière. Ces activités sont servies par les efforts entrepris en Grèce comme en Albanie dans les années 1990 pour combler le retard en équipements de l'espace frontière (voir carte). La collaboration avec les entrepreneurs grecs se solde chaque année, depuis 1999, par l'organisation d'une foire du commerce à Gjirokastër. Les entreprises FRESH, installée à Frashtan, ou Kuros, à Gjirokastër, qui fabrique des chaussures à façon pour le compte d'une entreprise italienne, ne pourraient pas exister sans la présence toute proche de la frontière ${ }^{31}$. En effet, la proximité de la Grèce permet à ces entrepreneurs de bénéficier

\footnotetext{
30 Voir par exemple le récit angoissé d'une traversée légale par le poste frontière de Kakavijë dans Kondi 2001, p. 16-18.

31 Ces deux entreprises ont été observées dans la région de Gjirokastër. La première est le produit d'une entente entre deux Grecs d'Albanie et un investisseur de Thessalonique, dès 1992. L'usine, qui emploie 80 ouvriers, est installée dans un village du Dropull et produit des boissons gazeuses qui sont distribuées dans tout le pays. L'entreprise détient également le monopole pour l'importation en Albanie de certains produits grecs. La seconde affaire est nettement plus modeste puisqu'elle a été créée par un émigré aroumain en 1999. Elle n'emploie aujourd'hui qu'une dizaine de personnes et ne fait que difficilement face aux désagréments de
} 
d'une source d'approvisionnement fiable pour un matériel professionnel qui leur fait défaut en Albanie alors que leur installation de ce côté-ci de la frontière est associée à l'emploi d'une main d'œuvre à très faible coût (300 lek [2,4 euros] par jour). Ces deux exemples sont révélateurs de la façon dont ce type d'activité met en jeu plusieurs types de risques. Le premier est de nature commerciale. Les deux entreprises se destinent au marché albanais et leur réussite est conditionnée par les capacités de celui-ci à fournir une offre régulière pour en absorber la production. D'autre part, le lien de dépendance étroite avec la Grèce nécessite de bonnes conditions de franchissement de la frontière entre les deux pays. De ce fait, l'appartenance à un groupe [264] reconnu par le gouvernement grec comme étant « d'origine grecque » est un atout indispensable et il n'est pas étonnant de constater que les deux chefs d'entreprise rencontrés sont, comme c'est le cas la plupart du temps dans la région de Gjirokastër, issus de la communauté hellénophone ou aroumaine, et bénéficient à ce titre de droits particuliers qui ne les astreignent pas à une demande de visa pour franchir la frontière. Ils sont en effet reconnus par la Grèce comme des étrangers d'origine grecque, ou omogeneis ("de même origine »), catégorie privilégiée par rapport à celles des allogeneis, les étrangers «d'une autre origine». "Je vais cinq fois par semaine à Ioannina pour y acheter des fournitures, explique le fondateur de Kuros. À Kakavijë, je connais tout le monde. Mon technicien qui est musulman ne peut pas venir avec moi. Il lui faudrait un visa chaque semaine et ce n'est pas possible de faire ça ». À cet atout indiscutable s'ajoute le fait que les omogeneis ont été les premiers à s'être rendus en Grèce et à y avoir accumulé un capital pouvant servir de base de départ à une affaire en Albanie.

Le développement d'initiatives privées sur cette partie de la zone frontalière apparait ainsi être aux mains de certains groupes (hellénophone et aroumain) qui valorisent de la sorte leurs rapports privilégiés avec la Grèce, alors que les autres - musulmans albanophones - n'y participent que dans la limite des tâches les moins valorisées. "Nous faisons venir nos ouvriers de Libohovë ou de Gjirokastër, précise le directeur de FRESH. Nous organisons leur trajet dans des autobus de la compagnie. Ce sont tous des musulmans. Cela nous revient moins cher que d'employer des ouvriers du village qui demandent plus d'argent ${ }^{32} »$. Ne doit-on pas voir dans ces disparités une des causes des tensions qui ont abouti aux violences dont ont fait l'objet les membres de la communauté hellénophone du Dropull en 1997 ? Mais cette différenciation liée à la proximité de la frontière connait d'autres implications que celles qui touchent au milieu des affaires.

\subsection{Frontières culturelles, frontières ethniques}

L'ouverture sur le monde extérieur et la multiplication des traversées et des échanges ne signifient pas pour autant l'homogénéisation de la société transfrontalière. On observe au contraire l'apparition ou la résurgence de limites entre des groupes définis sur différents

la situation générale du pays et surtout aux longues coupures d'électricité qui contraignent souvent l'usine au chômage technique.

32 Les salaires dans l'usine FRESH s'élèvent à 25000 lek (200 euros) par mois pour un ouvrier musulman, et à 40000 lek (320 euros) pour un hellénophone. 
critères, dans un processus de particularisation qui répond aux risques posés par la frontière.

La législation grecque entraîne l'apparition de clandestins qui traversent la frontière illégalement, hors des points de passage, ou avec de faux documents, ou encore qui séjournent en Grèce à l'expiration de leurs visas ou dans l'attente de documents définitifs. Apparaît alors une tension dans la zone frontalière [265] puisqu'elle est considérée, du côté albanais, mais surtout du côté grec, comme un lieu de passage ou de séjour pour des personnes jugées dangereuses en raison de l'illégalité de leur statut. Du côté grec, la population vit dans la peur de l'Albanais, qu'elle imagine errant dans les campagnes, voleur, violeur et assassin. Des groupes d'autodéfense peuvent dans certains cas se mettre en place. Le migrant albanais des premiers temps, le réfugié pauvre et misérable qu'il fallait secourir, a laissé la place à l'image du barbare, que l'on ne voit pas, mais que l'on sait rôder dans l'espace sauvage aux abords des villages (Seremetakis 1996). On lui attribue les trafics d'armes, de drogues et de prostituées qui l'associent à la criminalité organisée. On retrouve, dans ces discours, une forme de déshumanisation de l'autre dans les régions frontalières, qui n'est pas spécifique à la frontière gréco-albanaise (Donnan, Wilson 1999, p. 137-138). Cette tendance apparait également dans le comportement des forces de l'ordre envers les Albanais : aux points de passage et ailleurs, on ne leur parle pas, mais on leur hurle aux oreilles des instructions et des interdictions, on les fait se ranger, avancer, reculer, comme on le ferait avec un troupeau ${ }^{33}$. On peut se demander si les stéréotypes et la déshumanisation ne sont pas des réponses au risque de l'hétérogénéité culturelle, dans la mesure où ils reviennent à nier l'altérité culturelle au profit d'une altérité radicale de type nature/culture qui rejette les migrants du côté de la nature.

Toutefois, un sentiment d'insécurité comparable existe aussi du côté albanais, où la population locale voit d'un mauvais œil les candidats à l'émigration clandestine venus du reste du pays traverser la région ou y séjourner avant leur départ en Grèce. L'opposition entre la population frontalière et les autres est suffisamment forte pour perdurer dans le contexte migratoire, où les différents groupes de migrants ne se mélangent pas. Cela recoupe souvent une opposition nord/sud très forte en Albanie ainsi que des oppositions religieuses (de Rapper 2002a) ${ }^{34}$.

Par ailleurs, la politique de fermeture côté grec est un des facteurs de fragmentation et de différenciation de la société locale du coté albanais de la frontière. La discrimination positive à l'égard de certains Albanais (hellénophones principalement, mais aussi aroumanophones voire même une grande partie des chrétiens orthodoxes du pays) est un élément conduisant d'une part à la cristallisation des différenciations, de l'autre à la modification de la perception du territoire. Les appartenances religieuses, de même que l'association à un territoire ou à un groupe linguistique particulier déterminent en grande partie les chances des candidats à l'émigration. Elles deviennent [266] l'objet de négociations et de manipulation, comme lorsque des musulmans se font passer pour

33 C'est d'ailleurs en terme d'animalité que certains migrants évoquent leur condition : ils survivent comme des bêtes sauvages, chassés et pourchassés, obligés de dormir dehors et de se nourrir de fruits.

34 Voir aussi, dans un traitement littéraire, Graçi 1995. 
chrétiens, ou que des Albanais font valoir le mariage d'un membre de leur famille dans la minorité grecque pour se déclarer eux-mêmes Grecs d'Albanie. Elles tracent des limites entre différents groupes, plus ou moins favorisés en terme de migration: Grecs d'Albanie, Aroumains, Albanais chrétiens orthodoxes, musulmans, Çams (ce dernier n'étant pas reconnu officiellement en Grèce).

La position des différentes catégories de population par rapport à la minorité grecque d'Albanie, à la Grèce et aux revendications qu'on lui prête sur le Sud de l'Albanie devient par ailleurs un enjeu en matière de possibilité migratoire ou de traversée de la frontière. C'est ce qu'illustre l'histoire de ce musulman de Gjirokastër arrêté à Athènes en 1994 et qui se voit demander par le policier, devant une carte des Balkans, s'il place la frontière à Arta (version albanaise maximale) ou sur le Shkumbin (version grecque maximale), avant d'être relâché.

Il semble donc que le changement radical de statut de cette frontière ait eu un impact sur les appartenances collectives dans la région frontalière. Les identités régionales sont aussi un moyen de structurer la réalité devenue mouvante avec l'ouverture de la frontière. À une certaine échelle, cet impact se replace dans le processus général de reconfiguration identitaire dans l'Albanie postcommuniste avec la transformation des identités politiques, sociales, et la réaffirmation d'identités régionales ou confessionnelles, mais la région frontalière présente des spécificités.

Il peut en effet y avoir émergence d'un sentiment d'identité frontalière ou transfrontalière, avec les pratiques qui l'accompagnent. C'est le cas de certains villages du Pogon albanais, qui ne s'identifient à aucun des deux États : habitant en Albanie mais de langue et de conscience nationale grecque, les habitants du Pogon albanais ne se sentent chez eux que dans leur région frontalière. Leur sort a été marqué par l'établissement de la frontière, qui les a laissés en Albanie, alors que le reste du Pogon était attribué à la Grèce. Ils vivent aujourd'hui dans les deux pays : ils travaillent en Grèce, mais passent les vacances ou les fins de semaine en Albanie, transformant leurs maisons en résidences secondaires, phénomène encore assez rare en Albanie. Le point de passage dont ils disposent, récemment ouvert, est réservé aux piétons, les voitures ne peuvent traverser à cet endroit. Une organisation locale des transports s'est mise en place; elle comprend des parkings du côté grec, où on laisse sa voiture achetée et immatriculée en Grèce, et des taxis du côté albanais, souvent des véhicules à quatre roues motrices qui relient les villages au point de passage, sur des chemins de montagne. Le caractère enclavé de la zone, les conditions propres à la frontière dans cette région de l'Albanie et la condition de ses habitants participent à l'exacerbation de la situation de minorité dans le Pogon albanais.

[267] Autre configuration, celle des Albanais chrétiens orthodoxes, qui ont été et sont tiraillés entre l'appartenance à la nation albanaise et celle à la nation grecque. On observe ici l'émergence d'une identité locale, très territorialisée, centrée autour de la notion d'autochtonie. L'autochtonie permet de s'affirmer face à la minorité grecque, qui dans une certaine lecture de l'histoire est d'installation récente dans la région, face aussi aux Aroumains associés au nomadisme et que l'on dit être originaires de l' «autre côté » de la 
frontière. Face enfin aux Albanais musulmans qui, par le fait de la conversion à l'islam, acquièrent une origine étrangère. Les Albanais chrétiens développent ainsi une sorte de «nationalisme local» qui fait intervenir la langue (albanaise et non grecque ou aroumaine), la religion (chrétienne et non musulmane), un mythe d'origine (ils descendent des Chaones, tribu épirote occupant dans l'antiquité la vallée du Drino et le bassin de la Bistricë, valorisent le site antique d'Antigoné comme la métropole après la destruction de laquelle auraient été fondés les villages actuels), et un certain nombre de traits culturels (costumes, alimentation).

Les Albanais musulmans de la région sont confrontés à un autre problème: albanophones et musulmans, tout les éloigne de la Grèce vers laquelle ils sont pourtant très nombreux à migrer. Cette distance est diminuée dans la pratique par l'adoption d'un second prénom, chrétien, que l'on prend une fois passée la frontière et par différents degrés de conversion à l'orthodoxie (de Rapper 2002b, p. 209-211 et 221-225). C'est aussi en insistant sur le caractère de «mosaïque » de la région frontalière en Épire : l'Épire, dit-on, abrite de nombreux groupes différents (chrétiens, musulmans, Grecs, Valaques, Tsiganes, Çams,...), y compris celui des musulmans, dont la présence est ainsi justifiée. On projette dans le passé la plus lointain l’image de l'Épire comme «État multiethnique ». La valorisation de la fragmentation évite de se retrouver du mauvais côté de la frontière, qu'elle soit nationale ou religieuse, dans la mesure où, en les multipliant, elle relativise l'importance des frontières. Par ailleurs, les musulmans insistent sur leur origine chrétienne et sur les phénomènes de conversion, qui ont affecté tout le monde dans la région, dans les deux sens. Ils font donc eux-mêmes partie d'un phénomène qui ne les exclut pas, mais qui les intègre dans la région frontalière ${ }^{35}$.

Dernière configuration, celle des Aroumains, qui cultivent eux aussi une position de l'entre-deux. Se disant dans leur immense majorité originaires du côté grec de la frontière, où beaucoup d'entre eux sont effectivement nés, et où ils étaient désignés comme «Valaques albanais » (arvanitovlachoi) en raison de leur connaissance de la langue albanaise, acquise par la fréquentation de pâturages d'hiver situés du côté albanais, [268] ils ont été catégorisés comme "Grecs » en Albanie en raison de leur origine géographique, de leur absence de loyauté supposée envers l'État albanais et de leur remarquable hellénophonie. Pourtant, ils n'étaient pas reconnus comme minorité dans l'Albanie communiste (contrairement aux Grecs) et ont souffert de discrimination et de persécutions en tant que Valaques. Aujourd'hui, ils jouent sur cette origine grecque pour migrer plus facilement en Grèce, mais pas toujours de façon permanente. Ils finissent en effet par rencontrer les mêmes problèmes que les autres : ils sont Albanais, ou du moins viennent d'Albanie. Certains rentrent en Albanie, mais continuent de tirer profit de leur situation ambiguë (facilité à traverser la frontière); ils sont commerçants, entrepreneurs, souvent en collaboration avec des partenaires grecs. Ils sont par ailleurs les premiers dans la région à s'organiser en associations, avec une tendance à l'ethnicisme transnational décrit par Jean-

35 Il est curieux de voir qu'une telle conception rejoint celle de l'historien anglais Tom Winnifrith pour lequel l'Épire a de tout temps été une région frontière, dans laquelle les frontières ethniques n'ont cessé de se déplacer et d'être traversées (Winnifrith 2002). 
François Gossiaux (Gossiaux 2002, p. 186). Ils illustrent la valorisation de la mobilité, de la traversée et de l'interface.

\section{Conclusion}

Nous avons cherché à montrer en quoi l'apparition de la notion de risque sur la frontière gréco-albanaise a un impact sur la vie des populations frontalières. Le risque se présente sous deux aspects principaux : (1) risque de conflit international pouvant remettre en question la frontière elle-même et mettre en péril la sécurité des populations; (2) risque de rupture du lien entre l'État et les populations locales, prises dans un entrelacs de limites et d'allégeances diverses qui peuvent affaiblir leur loyauté. Les implications de la reconnaissance du risque frontalier par l'État touchent de près les populations frontalières, qu'il s'agisse de la militarisation de la zone frontière ou de la canalisation des sentiments d'appartenance par l'idéologie et la statistique. Les réponses étatiques entraînent à leur tour des réponses individuelles et collectives de la part des populations locales, qui s'expriment à la fois dans la pratique de la frontière et dans les processus de particularisation de certains groupes locaux.

Ces processus de particularisation renforcent l'hétérogénéité culturelle préexistante à l'établissement de la frontière, que nous avons identifiée comme l'un des aspects du risque frontalier. Dans le contexte européen de reconnaissance des minorités et de leurs droits collectifs, l'exacerbation de la particularisation peut elle-même donner lieu à des revendications sur la base du droit des minorités. Au jeu de différenciation et de particularisation induit par la frontière et ses usages s'ajoutent les effets de l'idéologie multiethnique véhiculée par les institutions et organisations internationales comme par les médias. En janvier 2002 a eu lieu le lancement d'un programme sur «les relations interethniques dans la région de Gjirokastër », initié par des journalistes et intellectuels, et soutenu financièrement par la fondation Soros. [269] Son objectif est « d'organiser des rencontres entre gens des différentes communautés - Grecs, Albanais, Valaques, Tsiganes - des journalistes, des hommes politiques et des fonctionnaires ${ }^{36} »$, alors que se multiplient dans la société albanaise les interrogations sur l'origine, l'histoire, le statut et le rôle des minorités. Les années 1990 ont par ailleurs été celles de l'apparition des Aroumains en tant que communauté organisée revendiquant une position particulière dans la société albanaise, ce qui a permis d'observer l'influence de la conception minoritaire en vigueur au niveau international sur le développement de sentiments d'appartenance au départ inscrits dans un contexte local. Des enquêtes ont également mis en évidence le rôle des États voisins (grec et roumain en l'occurrence) dans ces processus (Schwandner-Sievers 1998). Les relations internationales entre la Grèce et l'Albanie sont aussi marquées par la question des droits à reconnaitre de part et d'autre de la frontière, aux membres de la minorité grecque d'Albanie comme aux travailleurs albanais émigrés en Grèce. On peut se demander dans quelle mesure l'approche des relations transfrontalières en termes de droits des minorités contribue à une meilleure intégration de la région frontalière et si elle n'est pas au contraire

\footnotetext{
${ }^{36}$ D'après l'un des initiateurs du projet.
} 
un risque de segmentation des deux sociétés (Riedel 2002). Le cas de la frontière grécoalbanaise illustre en cela à la fois l'importance croissante des questions de minorités entre pays européens et le risque que ces dernières font peser sur l'intégrité territoriale des États.

\section{Bibliographie}

Barjaba, Kosta, 1995. Recent Implications of Interethnic Relations in Albania. Anthropological Journal on European Cultures 4, 1, p. 79-101.

Beci, Bahri, 1998. Les minorités ethniques en Albanie. Cabiers balkaniques 25, p. 19-26.

Belegu, Mentar, 1968. Mbi luftën e klasave në vendin tonë në vitet e para të çlirimit (1945-1947). Studime Historike V, 1, p. 189-199 (Sur la lutte des classes dans notre pays dans les premières années après la libération, 1945-1947).

Chiclet, Christophe, 2000. Les relations gréco-albanaises, amours ambiguës. Cabiers d'études sur la Méditerranée orientale et le monde turco-iranien 29, p. 225-232.

Clayer, Nathalie, 2004. Frontière politique, frontière ethnique et État-nation. L'exemple de la région-frontière albano-grecque dans l'entre-deux-guerres. in D. Necak (dir.), Borders in Southeastern Europe: Culture and Politics between the 18th and 21th Century. Ljubljana, University of Ljubljana. p. 159-175.

Daniel, Odile, 1996. L’Albanie libérée des légendes. in Europe orientale, Russie, Asie Centrale. Paris, Belin Reclus.

de Rapper, Gilles, 1997. Les villages de Kastoria ou la minorité imaginée. Sighrona Themata 63, p. 108-111 (en grec).

de Rapper, Gilles, 2002a. Grenzen überschreiten: Migration in der albanischen Grenzregion Devoll. in K. Kaser, R. Pichler and S. Schwandner-Sievers (dir.), Die weite Welt und das Dorf. Albanische Emigration am Ende des 20. Jabrbunderts. Wien-Köln-Weimar, Bölhau. p. 83-106 (Traverser la frontière : la migration dans une région frontalière albanaise, le Devoll).

de Rapper, Gilles, 2002b. Transformation und Anpassung: Die albanischen Zuwander in Griechenland zwischen Integration und Rassismus. in U. Brunnbauer (dir.), Umstrittene Identitäten. Ethniəiität und Nationalität in Südosteuropa. Frankfurt am Main, Peter Lang. p. 201-232 (Transformation et adaptation : les migrants albanais en Grèce, entre intégration et racisme).

de Rapper, Gilles, 2004. 'We are not Greek, but...': Dealing with the Greek-Albanian Border among Albanian-speaking Christians of Southern Albania. Journal of Southeast European and Black Sea Studies 4, 1, p. 162-174. 
de Rapper, Gilles, 2005. Better than Muslims, not as good as Greeks: emigration as experienced and imagined by the Albanian Christians of Lunxhëri. in R. King, N. Mai and S. Schwandner-Sievers (dir.), The New Albanian Migration. Brighton-Portland, Sussex Academic Press. p. 173-194.

de Rapper, Gilles, Olivier Deslondes and Michel Roux, 2000. Dimanche à Miras, lundi à Dipotamia. La frontière albano-grecque dans la région de Bilisht et de Kastoria. Cahiers d'études sur la Méditerranée orientale et le monde turco-iranien 29, p. 199-223.

Deslondes, Olivier, 1997. Les fourreurs de Kastoria, entre la Macédoine et l'Occident. Paris, CNRS Editions.

Donnan, Hastings and Thomas M. Wilson, 1999. Borders: Frontiers of Identity, Nation and State. London, Berg.

Febvre, Lucien, 1928. Frontière : le mot et la notion. Bulletin du Centre International de Synthèse XLV, p. 31-44.

Gossiaux, Jean-François, 2002. Pouvoirs ethniques dans les Balkans. Paris, PUF.

Graçi, Virion, 1995. Të çmendur në parajsë. Tiranë, Dituria (traduction française par C. Montécot : Virion Graçi, Le paradis des fous, La Tour-d'Aigues, 1998, réédition Gallimard, 2000).

Green, Sarah, 2005. Notes from the Balkans. Locating Marginality and Ambiguity on the Greek-Albanian Border. Princeton, Princeton University Press.

Hoxha, Enver, 1985. Deux peuples amis. Tirana, 8 Nëntori.

Hoxha, Ibrahim D., 2000. Viset kombëtare shqiptare në shtetin grek. Tiranë, Hasan Tahsini (Les régions nationales albanaises dans l'État grec).

Jandot, Gabriel, 1994. L'Albanie d'Enver Hoxba (1944-1985). Paris, L'Harmattan.

Kadaré, Ismail, 2002. Vie, jeu et mort de Lul Marrek. Paris, Fayard.

Kahl, Thede, 2002. The Ethnicity of Aromanians after 1990: the Identity of a Minority that behaves like a Majority. Ethnologia Balkanica 6, p. 145-169.

Kasoruho, Amik, 1996. Një ankth gijsmëshekullor. Shqipëria e Enver Hoxbës. Tiranë, Çabej (Une peur demiséculaire. L'Albanie d'Enver Hoxha).

Kondi, Arben, 2001. Rikthim në Camëri. Tiranë, ILAR (Retour en Çamëri). 
Kondis, Basil and Eleftheria Manda, 1994. The Greek Minority in Albania. A Documentary Record (1921-1993). Thessaloniki, Institute for Balkan Studies.

Konomi, Vasil, 2001. Greqia - Shqipëria dhe emigranti. Tiranë, GEER (La Grèce, l'Albanie et le migrant).

Kretsi, Georgia, 2002. The 'Secret' Past of the Greek-Albanian Borderlands, Cham Muslim Albanians: Perspectives on a Conflict over Historical Accountability and Current Rights. Ethnologia Balkanica 6, p. 171-195.

Madhi, Sotir, 1967. Lufta e PPSH kundër oportunizmit të djathtë në vitet 1949-1955. Studime Historike IV, 3, p. 43-58 (La lutte du PTA contre l'opportunisme de droite pendant les années 1949-1955).

Puto, Arben, 1974. Qeveria demokratike e 1924-ës për zgjidhjen e problemeve të mbetura pezull në caktimin e kufijve të Shqipërisë. Studime Historike XI, 2, p. 87-110 (Le gouvernement démocratique de 1924 et la résolution des problèmes demeurés en suspens dans la délimitation des frontières de l'Albanie).

Riedel, Sabine, 2002. Minorités nationales en Europe et protection des droits de l'homme : un enjeu pour l'élargissement. Politique étrangère 3, p. 647-664.

Schwandner-Sievers, Stephanie, 1998. Ethnicity and Transition: The Albanian Aromanians' Identity Politics. Ethnologia Balkanica 2, p. 167-184.

Seremetakis, C. Nadia, 1996. In Search of the Barbarians: Borders in Pain. American Anthropologist 98, 3, p. 489-491.

Sintès, Pierre, 2003. Les Albanais en Grèce. Le rôle des réseaux préexistants. Balkanologie VII, 1, p. 111-133.

Sivignon, Michel, 1989. Ruptures et reclassements dans l'Épire grecque. Espace rural 20, p. 61-85.

Tsitselikis, Constantin and Dimitris Christopoulos, 2003. La minorité grecque d'Albanie. Athènes, KEMO (en grec).

Winnifrith, Tom. J., 2002. Badlands - Borderlands. A History of Southern Albania / Northern Epirus. London, Duckworth.

Woodhouse, Christopher M., 2002. The Struggle for Greece, 1941-1949. London, Hurst.

Zarrilli, Luca, 1999. Albania, geografia della transiz̨ione. Milan, Francoangeli (Albanie, géographie de la transition). 